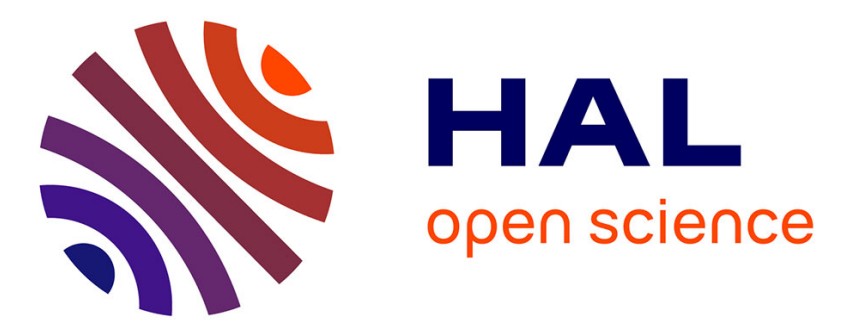

\title{
Stability analysis of a steady state of a model describing Alzheimer's disease and interactions with prion proteins
}

Mohammed Helal, Angélique Igel-Egalon, Abdelkader Lakmeche, Pauline

Mazzocco, Angélique Perrillat-Mercerot, Laurent Pujo-Menjouet, Human

Rezaei, Léon Matar Tine

\section{To cite this version:}

Mohammed Helal, Angélique Igel-Egalon, Abdelkader Lakmeche, Pauline Mazzocco, Angélique Perrillat-Mercerot, et al. Stability analysis of a steady state of a model describing Alzheimer's disease and interactions with prion proteins. Journal of Mathematical Biology, 2019, 78 (1-2), pp.57-81. 10.1007/s00285-018-1267-1 . hal-01946610

\section{HAL Id: hal-01946610 https://hal.science/hal-01946610}

Submitted on 7 Dec 2018

HAL is a multi-disciplinary open access archive for the deposit and dissemination of scientific research documents, whether they are published or not. The documents may come from teaching and research institutions in France or abroad, or from public or private research centers.
L'archive ouverte pluridisciplinaire HAL, est destinée au dépôt et à la diffusion de documents scientifiques de niveau recherche, publiés ou non, émanant des établissements d'enseignement et de recherche français ou étrangers, des laboratoires publics ou privés. 


\title{
Stability Analysis of a Steady State of a Model Describing Alzheimer's Disease and Interactions with Prion Proteins
}

\author{
Mohammed Helal • Angélique Igel-Egalon • \\ Abdelkader Lakmeche • Pauline Mazzocco . \\ Angélique Perrillat-Mercerot • Laurent \\ Pujo-Menjouet · Human Rezaei · Léon M. Tine
}

Received: date / Accepted: date

\begin{abstract}
Alzheimer's disease (AD) is a neuro-degenerative disease affecting more than 46 million people worldwide in 2015. AD is in part caused by the accumulation of $\mathrm{A} \beta$ peptides inside the brain. These can aggregate to form insoluble oligomers or fibrils. Oligomers have the capacity to interact with neurons via membrane receptors such as prion proteins $\left(\mathrm{PrP}^{\mathrm{C}}\right)$. This interaction leads $\mathrm{PrP}^{\mathrm{C}}$ to be misfolded in oligomeric prion proteins $\left(\mathrm{PrP}^{\mathrm{ol}}\right)$, transmitting a death signal to neurons.

In this work, we propose a new mathematical model bringing together different mechanisms: $\mathrm{A} \beta$ polymerization, including formation of oligomers and fibrils, and interaction between $\mathrm{A} \beta$ oligomers and prion proteins. The model is based on Becker-Döring equations for the polymerization process, with delayed differential equations accounting for $\mathrm{A} \beta / \mathrm{PrP}^{\mathrm{C}}$ interactions. We analyse the well-posedness of the model and show existence, uniqueness and non-negativity of
\end{abstract}

This work has been supported by Association France-Alzheimer, SM 2014.

M. Helal

University Sidi Bel Abbes, Laboratory of Biomathematics, Sidi Bel Abbes, Algeria

A. Igel-Egalon

INRA, UR892 Virologie Immunologie Moléculaires, 78352 Jouy-en-Josas, France

A. Lakmeche

University Sidi Bel Abbes, Laboratory of Biomathematics, Sidi Bel Abbes, Algeria

P. Mazzocco

Université de Lyon, Université Claude Bernard Lyon 1, CNRS UMR 5558, Laboratoire de Biométrie et Biologie Evolutive, 69100 Villeurbanne, France

A. Perrillat-Mercerot

Université de Poitiers, Laboratoire de Mathématiques et Applications, UMR CNRS 7348, SP2MI Equipe DACTIM-MIS, F-86962 Chasseneuil Futuroscope Cedex, France

L. Pujo-Menjouet, corresponding author

Université de Lyon, Université Claude Bernard Lyon 1, CNRS UMR 5208, Institut Camille Jordan, F-69622

Villeurbanne cedex; Inria Team Dracula, Inria Grenoble Rhône-Alpes Center, 69100 Villeurbanne, France

E-mail: pujo@math.univ-lyon1.fr

H. Rezaei

INRA, UR892 Virologie Immunologie Moléculaires, 78352 Jouy-en-Josas, France

L.M. Tine

Université de Lyon, Université Claude Bernard Lyon 1, CNRS UMR 5208, Institut Camille Jordan, F-69622

Villeurbanne cedex; Inria Team Dracula, Inria Grenoble Rhône-Alpes Center, 69100 Villeurbanne, France 
solutions. Moreover, we demonstrate that this model admits a non-trivial steady state, which is found to be globally stable thanks to a Lyapunov function. We finally present numerical simulations and discuss the impact of model parameters on the whole dynamics, which could constitute the main targets for pharmaceutical industry.

Keywords mathematical model analysis $\cdot$ steady state $\cdot$ Alzheimer's Disease $\cdot$ prions $\cdot$ numerical simulations

Mathematics Subject Classification (2010) 34D23 - 92B05

\section{Introduction}

\section{Biological background}

Alzheimer's disease (AD) is a fatal incurable disease known as the most common form of dementia (60 to $80 \%$ of dementia cases (Sosa-Ortiz et al., 2012)). Affecting more than 46 million people worldwide in 2015 (Prince et al., 2015), it causes progressive neuron degeneration, leading to loss of mental functions such as memory, language or behaviour. Although AD arises most of the time in the elderly community, this disease is not considered as a normal consequence of ageing. Causes of Alzheimer's disease remain uncertain. Different hypotheses, such as cholinergic (Francis et al., 1999), amyloid or Tau hypotheses, have been proposed to explain AD appearance. The two last hypotheses seem to be currently the most plausible ones. Indeed, accumulation of amyloid plaques inside the brain (Karran et al., 2011) and abnormalities of Tau protein are observed in diseased patients (Maccioni et al., 2010). Although these two processes could evolve simultaneously (Small and Duff, 2008), it seems that the first biomarkers becoming abnormal in $\mathrm{AD}$ are the concentrations of amyloid $\mathrm{A} \beta-40$ and $\mathrm{A} \beta-42$ in patient cerebrospinal fluid (Jack et al., 2013). A $\beta$ monomers are obtained from a specific cleavage of the amyloid precursor protein APP, leading to their release and accumulation inside the brain (Nunan and Small, 2000). The major isoforms are composed of 39 to 43 amino acids, $\mathrm{A} \beta$ - 40 being the most common type of monomers, and $\mathrm{A} \beta-42$ a plausible evidence of Alzheimer's disease (Bitan et al., 2003; Johnson et al., 2013)). A $\beta$ monomers could at least follow two distinct polymerization pathways. The first one corresponds to the fibrillation pathway well described by the canonical nucleation elongation process (Lomakin et al., 1996) with the particularity that $\mathrm{A} \beta$ fibrils have been reported to be able to depolymerize (Carulla et al., 2005) rendering each step of the fibrils elongation process reversible (Figure 1, blue part). The second pathway corresponds to the $\mathrm{A} \beta$ oligomerization pathway. As for fibrillation, the oligomerization process follows a nucleation elongation mechanism with the difference that the oligomerization pathway leads to the formation of highly stable $\mathrm{A} \beta$ assemblies structurally distinct from A $\beta$ fibrils (Nick et al. (2018); Barz et al. (2017)). Regarding the literature, several neurotoxic pathways not mutually exclusive and all involving specifically A $\beta$ oligomers have been proposed (Kessels et al. (2010); James (2013); Kandel et al. (2017)). Recently one of these pathways, involving the binding of $\mathrm{A} \beta$ oligomers to prion protein $\operatorname{PrP}^{\mathrm{C}}$ under its non-pathogenic monomeric conformer, has been reported to be involved in a deathsignal transduction into the neurons through the oligomerization of $\operatorname{PrP}^{\mathrm{C}}$ (Kessels et al. (2010); Gimbel et al. (2010)). Even if the molecular mechanisms of this transduction signal are not yet clear, the oligomerization of $\operatorname{PrP}^{\mathrm{C}}$ (named here $\mathrm{PrP}^{\mathrm{ol}}$ ) has been reported to be involved in the dead signal transduction (Cissé and Mucke (2009); Laurén et al. (2009); Gimbel et al. (2010)). In term of physical-chemistry, the interaction between $\operatorname{PrP}^{\mathrm{C}}$ and $\mathrm{A} \beta$ oligomers leading to $\operatorname{PrP}^{\mathrm{ol}}$ formation could be decomposed into two steps: the first step corresponds to the formation of $\mathrm{PrP}^{\mathrm{C}} / \mathrm{A} \beta$ complex (Freir et al. (2011); Gallion (2012)). Then after a delay (denoted $\tau$ in this work), corresponding to a structural rearrangement (Hilser et al., 2012), the complex evolves to 
generate $\mathrm{PrP}^{\mathrm{C}}$ oligomerization.

\section{Mathematical background}

These processes are challenging for biologists. Indeed, it is not easy to explain the observed kinetic behaviours in experiments. Therefore an interdisciplinary collaboration between mathematicians and biologists lead to a new mathematical model where these considerations have been discussed and supported by biological assumptions and observations.

While a number of studies focus either on aggregation of $\mathrm{A} \beta$ monomers (see for instance Lomakin et al. (1997); Urbanc et al. (1999); Craft (2002); Achdou et al. (2013); Bertsch et al. (2016)) or on $\mathrm{PrP}^{\mathrm{C}}$ proliferation (Greer et al. (2006); Prüss et al. (2006); Engler et al. (2006); Calvez et al. (2009); Gabriel (2011), to cite a few), to the best of our knowledge, only two mathematical models have been proposed to study $\mathrm{A} \beta / \mathrm{PrP}^{\mathrm{C}}$ interactions (Helal et al., 2014; Ciuperca et al.). In the first one, authors describe plausible in vivo interactions between $\mathrm{A} \beta$ oligomers and $\operatorname{PrP}^{\mathrm{C}}$, but did not focus on the whole process of polymerization (Helal et al., 2014). In the second article, authors propose a complex model including $\mathrm{A} \beta$ polymerization and interactions with prion (Ciuperca et al.). However, this model assumes a continuous size for polymers, a hypothesis generally made for large objects, but which can be controversial for oligomers. Besides, because the model is based on partial differential equations, its analysis can be more complicated.

\section{Objectives of this work}

We aim herein at building a model describing in vitro polymerization process of $\mathrm{A} \beta$ monomers in fibrils or oligomers, and interactions with prion proteins, mechanisms that seem to initiate development of Alzheimer's disease. We base our model on Becker-Döring equations (Becker and Döring, 1935), assuming therefore a discrete size of polymers.

Our paper is organized as follows: we first present the mathematical model proposed to describe the different mechanisms of interest. We then investigate its well-posedness and show that the model admits a unique and globally stable steady state. We finally present some numerical simulations and study the impact of model parameters on the whole dynamics.

\section{Mathematical modelling}

\subsection{Description and notations}

Our model is built in an in vitro context, as most experimental data are obtained in vitro. This means that no source or loss terms of any proteins involved in the system are considered. As a consequence, the total mass of the system should remain constant. We study evolution of $\mathrm{A} \beta$ monomers seeded at time $t=0$ in an environment containing $\operatorname{PrP}^{\mathrm{C}}$.

The model consists of two main parts: 1) polymerization process, including formation of oligomers and fibrils and 2) interactions between oligomers and prion (Figure 1).

During polymerization process, monomers can either form fibrils or proto-oligomers. These can then grow by attaching a monomer, or lose one at a rate $b_{f}$ for fibrils and $b$ for proto-oligomers. Rates of polymerization depend on polymer size. Indeed, we assume that longer polymers have more probability to polymerize. Proto-oligomers can reach a maximal size $i_{0}$, at which they become stable and can neither polymerize nor depolymerize. They then are called oligomers. A small proportion $\mu$ of oligomers are still able to split into two proto-oligomers of sizes $i$ and $i_{0}-i$. This process is not represented in Figure 1 for clarity. A $\beta$ oligomers can then be transported to the amyloid plaque at a rate $\gamma$ or form a complex with $\operatorname{PrP}^{\mathrm{C}}$ at a rate $\delta$. This interaction takes an incompressible time $\tau$ during which $\mathrm{PrP}^{\mathrm{C}}$ is misfolded into $\mathrm{PrP}^{\mathrm{ol}}$. The oligomer is released 


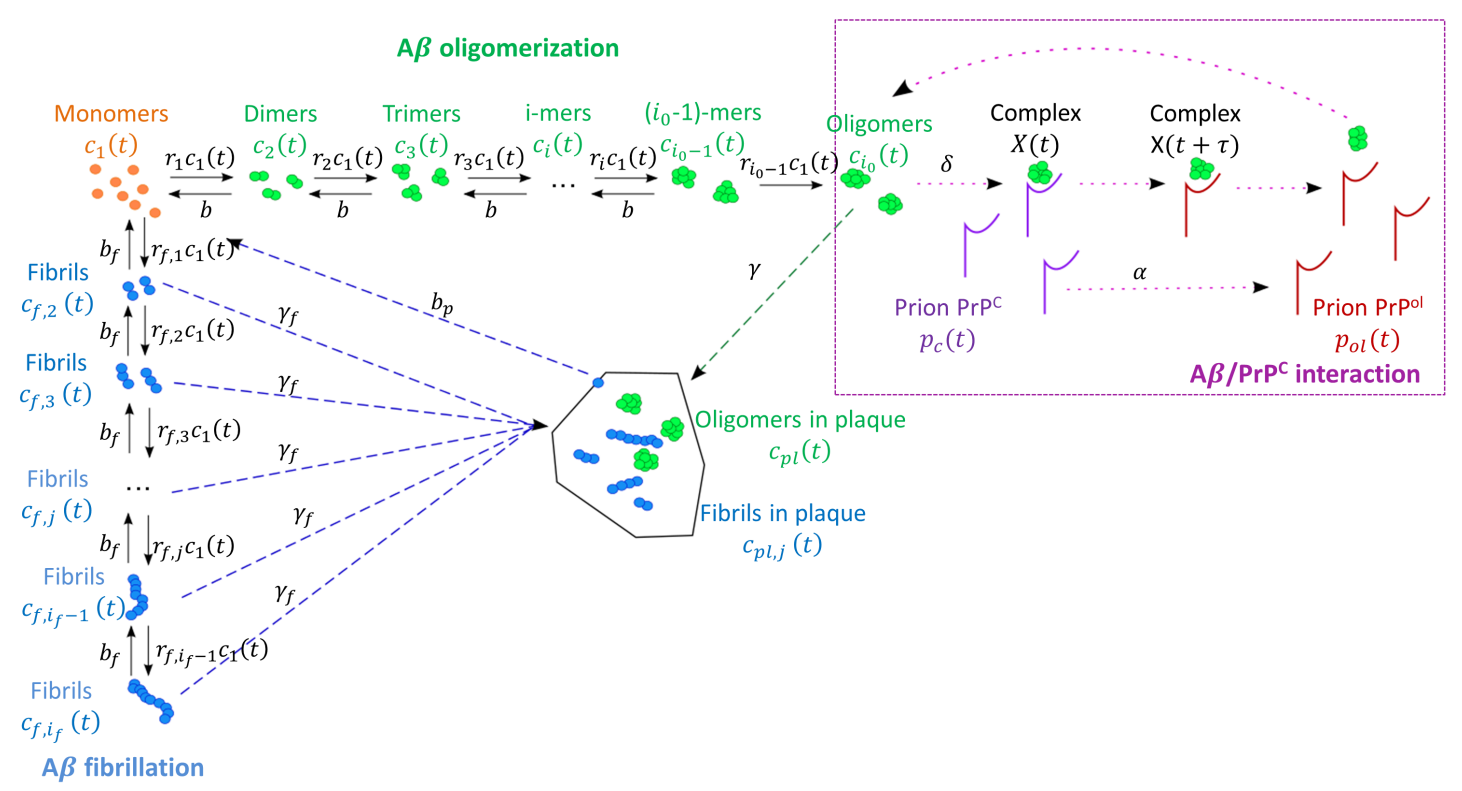

Fig. 1: Schematic representation of $\mathrm{A} \beta$ polymerization processes and interactions with $\mathrm{PrP}^{\mathrm{C}}$ prions. Orange part corresponds to monomer evolution. Blue part is related to fibrillation process, while green part is related to oligomerization part. Finally pink box (top right corner) corresponds to $\mathrm{A} \beta / \mathrm{PrP}^{\mathrm{C}}$ interaction. All parameters, quantities and interactions are described in the main text.

intact from the complex and can interact with another prion or be transported to the plaque. We also add the possibility for $\operatorname{PrP}^{\mathrm{C}}$ to directly transform into $\mathrm{PrP}^{\text {ol }}$ at a rate $\alpha$. This process can include other misfolding mechanisms that we are not aware of.

We assume that there is no fibril of infinite size, and therefore set $i_{f}$ as the maximal size they can reach. Fibrils of each size are also transported in the amyloid plaque at a rate $\gamma_{f}$. As they are still able to depolymerize, monomers can escape the plaque at a rate $b_{p}$. All notations and parameters are reported in Table 1 along with their description. 


\begin{tabular}{ll}
\hline Parameter/Variable & Definition \\
\hline Monomer & \\
\hline$c_{1}(t)=c_{f, 1}(t)$ & $\mathrm{A} \beta$ monomer concentration \\
\hline Oligomerization & Maximal size of A $\beta$ proto-oligomers \\
\hline$i_{0}$ & Size $i$ proto-oligomer concentration \\
$c_{i}(t), i \in\left\{2, \cdots, i_{0}-1\right\}$ & $\mathrm{A} \beta$ oligomer and oligomer in plaque concentrations \\
$c_{i_{0}}(t), c_{p l}(t)$ & Polymerization rate of size $i$ proto-oligomers (or monomers) \\
$r_{i}, i \in\left\{1, \cdots, i_{0}-1\right\}$ & Depolymerization rate of proto-oligomers \\
$b$ & Displacement rate of A oligomers into the plaque \\
$\gamma$ & Fragmentation kernel for oligomers \\
$K_{i_{0}, i}$ & Proportion of oligomer fragmentation \\
$\mu$ & \\
\hline $\mathbf{F i b r i l l a t i o n}$ & Maximal size of A $\beta$ fibrils \\
\hline$i_{f}$ & Size $i$ fibril and fibril in plaque concentrations \\
$c_{f, i}(t), c_{p l, i}(t), i \in\left\{2, \cdots, i_{f}\right\}$ & Polymerization rate of size $i$ fibrils $($ or monomers) \\
$r_{f, i}, i \in\left\{1, \cdots, i_{f}-1\right\}$ & Depolymerization rates of fibrils and fibrils in plaque \\
$b_{f}, b_{p}$ & Displacement rate of A $\beta$ fibrils into the plaque \\
$\gamma_{f}$ & \\
\hline $\mathbf{A} \beta / \mathbf{P r P C}$ interaction & PrPC PrPol and A $\beta /$ PrPC complex concentrations \\
\hline$p_{c}(t), p_{o l}(t), X(t)$ & Reaction rate between A $\beta$ oligomers and PrP \\
$\delta$ & Duration of A $\beta$ and PrP interaction \\
$\tau$ & Rate of direct transformation of PrP into PrPol \\
$\alpha$ &
\end{tabular}

Table 1: Description of model variables and parameters.

2.2 Mathematical equations

Based on Becker-Döring equations (Becker and Döring, 1935), the first part of our model is as follows:

\section{Monomers}

$$
\begin{aligned}
c_{1}^{\prime}(t)= & -2\left(r_{1} c_{1}(t)^{2}-b c_{2}(t)\right)-\sum_{i=2}^{i_{0}-2}\left(r_{i} c_{i}(t) c_{1}(t)-b c_{i+1}(t)\right)-r_{i_{0}-1} c_{i_{0}-1}(t) c_{1}(t) \\
& -2\left(r_{f, 1} c_{1}(t)^{2}-b_{f} c_{f, 2}(t)\right)-\sum_{j=2}^{i_{f}-1}\left(r_{f, i} c_{f, i}(t) c_{1}(t)-b_{f} c_{f, i+1}(t)\right) \\
& +2 b_{p} c_{p l, 2}+\sum_{j=2}^{i_{f}-1} b_{p} c_{p l, i+1}(t)
\end{aligned}
$$

Proto-oligomers $\left(i \in\left\{2, \cdots, i_{0}-2\right\}\right)$

$c_{i}^{\prime}(t)=r_{i-1} c_{i-1}(t) c_{1}(t)+b c_{i+1}(t)-r_{i} c_{i}(t) c_{1}(t)-b c_{i}(t)+\mu K_{i_{0}, i} c_{i_{0}}(t)$,

$c_{i_{0}-1}^{\prime}(t)=r_{i_{0}-2} c_{i_{0}-2}(t) c_{1}(t)-r_{i_{0}-1} c_{i_{0}-1}(t) c_{1}(t)-b c_{i_{0}-1}(t)$,

\section{Oligomers}

$c_{i_{0}}^{\prime}(t)=r_{i_{0}-1} c_{i_{0}-1}(t) c_{1}(t)-\left(\gamma+\frac{\mu}{2}\right) c_{i_{0}}(t)-\delta c_{i_{0}}(t) p_{c}(t)+\delta c_{i_{0}}(t-\tau) p_{c}(t-\tau)$,

\section{Oligomers in plaque}

$c_{p l}^{\prime}(t)=\gamma c_{i_{0}}(t)$,

Fibrils $\left(j \in\left\{2, \cdots, i_{f}-1\right\}\right)$

$c_{f, j}^{\prime}(t)=r_{f, j-1} c_{f, j-1}(t) c_{1}(t)+b_{f} c_{f, j+1}(t)-r_{f, j} c_{f, j}(t) c_{1}(t)-b_{f} c_{f, j}(t)-\gamma_{f} c_{f, j}(t)$,

$c_{f, i_{f}}^{\prime}(t)=r_{f, i_{f}-1} c_{f, i_{f}-1}(t) c_{1}(t)-b_{f} c_{f, i_{f}}(t)-\gamma_{f} c_{f, i_{f}}(t)$,

Fibrils in plaque $\left(j \in\left\{2, \cdots, i_{f}-1\right\}\right)$

$c_{p l, j}^{\prime}(t)=b_{p} c_{p l, j+1}(t)-b_{p} c_{p l, j}(t)+\gamma_{f} c_{f, j}(t)$,

$c_{p l, i_{f}}^{\prime}(t)=-b_{p} c_{p l, i_{f}}(t)+\gamma_{f} c_{f, i_{f}}(t)$, 
with $t$ in $[\tau,+\infty)$ for equation (4) and $t$ in $[0,+\infty)$ for others. For $t$ in $[0, \tau)$, equation (4) becomes:

$$
c_{i_{0}}^{\prime}(t)=r_{i_{0}-1} c_{i_{0}-1}(t) c_{1}(t)-\left(\gamma+\frac{\mu}{2}\right) c_{i_{0}}(t)-\delta c_{i_{0}}(t) p_{c}(t) .
$$

Initial conditions are the following:

$$
\begin{cases}c_{1}(t=0)=m>0, & \\ c_{i}(t=0)=0, & i \in\left\{2, \cdots, i_{0}\right\}, \\ c_{p l}(t=0)=0, & \\ c_{f, j}(t=0)=0, & j \in\left\{2, \cdots, i_{f}\right\}, \\ c_{p l, j}(t=0)=0, & j \in\left\{2, \cdots, i_{f}\right\},\end{cases}
$$

which means that there are only monomers initially.

Equation (1) describes $\mathrm{A} \beta$ monomer evolution (orange part in Figure 1), obtained by computing monomer gain and loss from every proto-oligomer and fibril. Equation (2) governs proto-oligomer behaviour (green part in Figure 1). Proto-oligomers of size $i$ come from proto-oligomers of size $i-1$ that have attached a monomer (first term in equation (2)) or from proto-oligomers of size $i+1$ that have lost a monomer (second term in equation (2)). Similarly, proto-oligomers of size $i$ can attach or lose a monomer, and leave this compartment (third and fourth terms in equation (2)). The last term in equation (2) describes the fragmentation process: oligomers can divide in two proto-oligomers of size lower than $i_{0}-1$. An extra equation is needed for proto-oligomers of size $i_{0}-1$ (equation (3)) as oligomers do not depolymerize and consequently no proto-oligomers of size $i_{0}-1$ come from an oligomer. Equation (4) describes oligomer evolution. Delayed terms express oligomer release from a complex created $\tau$ units of time before. Finally, equation (5) describes the evolution of oligomers in plaque while equations (6)-(7) (respectively equations (8)-(9)) correspond to fibril dynamics (respectively fibrils in plaque) (blue part in Figure 1).

The second part of the model concerns $\mathrm{A} \beta$ oligomers and $\mathrm{PrP}^{\mathrm{C}}$ interactions (pink box in Figure 1):

$$
\begin{aligned}
& p_{c}^{\prime}(t)=-\delta c_{i_{0}}(t) p_{c}(t)-\alpha p_{c}(t), \\
& p_{o l}^{\prime}(t)=\delta c_{i_{0}}(t-\tau) p_{c}(t-\tau)+\alpha p_{c}(t), \\
& X^{\prime}(t)=\delta c_{i_{0}}(t) p_{c}(t)-\delta c_{i_{0}}(t-\tau) p_{c}(t-\tau),
\end{aligned}
$$

with $t$ in $[0,+\infty)$ for equation (12) and $t$ in $[\tau,+\infty)$ for equations (13)-(14).

Evolution of prion concentration is given by equation (12): $\operatorname{PrP}^{\mathrm{C}}$ prions either form a complex with $\mathrm{A} \beta$ oligomer or directly transform into $\mathrm{PrP}^{\mathrm{ol}}$. Equations (13) and (14) describe evolution of $\mathrm{PrP}^{\mathrm{ol}}$ and complexes.

On $[0, \tau)$, equations $(13)-(14)$ become:

$$
\begin{aligned}
p_{o l}^{\prime}(t) & =\alpha p_{c}(t), \\
X^{\prime}(t) & =\delta c_{i_{0}}(t) p_{c}(t),
\end{aligned}
$$

and initial conditions are the following:

$$
\left\{\begin{array}{l}
p_{c}(t=0)=p>0 \\
p_{o l}(t=0)=0 \\
X(t=0)=0
\end{array}\right.
$$

meaning that only $\operatorname{PrP}^{\mathrm{C}}$ are present initially. 
We should now verify whether the total mass remains constant over time. We can actually divide this mass in two: $\mathrm{A} \beta$ polymer mass $Q$ and prion mass $Q_{p}$ :

$$
\begin{aligned}
& Q(t)=c_{1}(t)+\sum_{i=2}^{i_{0}} i c_{i}(t)+\sum_{j=2}^{i_{f}} j\left(c_{f, j}(t)+c_{p, j}(t)\right)+i_{0}\left(c_{p l}(t)+X(t)\right), \\
& Q_{p}(t)=m_{p}\left(p_{c}(t)+p_{o l}(t)+X(t)\right) .
\end{aligned}
$$

Derivatives of these functions should be equal to zero:

$$
\begin{aligned}
& Q^{\prime}(t)=\mu c_{i_{0}}(t)\left(\sum_{i=2}^{i_{0}-2} i K_{i_{0}, i}-\frac{i_{0}}{2}\right), \\
& Q_{p}^{\prime}(t)=0 .
\end{aligned}
$$

Therefore, the fragmentation kernel should satisfy the following relation:

$$
2 \sum_{i=2}^{i_{0}-2} i K_{i_{0}, i}=i_{0} .
$$

This condition describes the fact that the sum of all successive fragment sizes should be equal to the initial size. Note that an oligomer can fragment either in proto-oligomers of sizes $i$ and $i_{0}-i$ or in proto-oligomers of sizes $i_{0}-i$ and $i$. These two possibilities are taken into account thanks to the factor 2 .

For instance, the following fragmentation kernel verifies relation (18) and guarantees mass conservation with time:

$$
K_{i_{0}, i}=\frac{1}{i_{0}-3}, \quad i \in\left\{2, \cdots, i_{0}-2\right\}
$$

This fragmentation kernel follows an uniform distribution, meaning that all fragmentations have the same probability to occur. As an example, this kernel has been used to describe the splitting of prion fibrils in (Prüss et al., 2006).

\section{Model analysis}

In this section, we focus on the mathematical analysis of the model. We first present classical results of existence, uniqueness and non-negativity of solutions. We then prove the existence of a unique and non-trivial steady state. Besides, with a Lyapunov function, we show that it is globally stable, which constitutes our main result.

3.1 Existence, uniqueness and non-negativity of solutions

Proposition 1 System of equations (1)-(14) admit a unique solution on $[0,+\infty)$. Besides, provided that the initial conditions are non-negative, the solution remains non-negative.

Proof We prove existence and uniqueness of solutions with the method of steps: we start by proving existence, uniqueness and non-negativity on $[0, \tau)$, then on $[\tau, 2 \tau)$, and extend these results on intervals $[n \tau,(n+1) \tau)$, for all $n$ in $\mathbb{N}$. 
Let us note, for all $t$ in $[0,+\infty)$ :

$$
\begin{aligned}
Z(t)= & { }^{\mathbf{t}}\left[c_{1}(t), c_{2}(t), \cdots, c_{i_{0}}(t), c_{p l}(t), c_{f, 2}(t), \cdots, c_{f, i_{f}}(t), c_{p l, 2}(t), \cdots, c_{p l, i_{f}}(t),\right. \\
& \left.p_{c}(t), p_{o l}(t), X(t)\right] .
\end{aligned}
$$

On $[0, \tau)$, the system of equations (1)-(14) can be written as $Z^{\prime}(t)=H_{1}(t, Z(t))$, with $Z(0)=$ $\left[m, c_{2}(0)=0, \cdots, c_{p l, i_{f}}(0)=0, p, 0,0\right] . H_{1}$ is a polynomial function given by:

$$
H_{1}(Z)=\left(\begin{array}{c}
H_{1,1}(Z) \\
r_{1} Z_{1}^{2}-b Z_{2}-r_{2} Z_{2} Z_{1}+b Z_{3}+\mu K_{i_{0}, 2} Z_{i_{0}} \\
\vdots \\
r_{i-1} Z_{i-1} Z_{1}-b Z_{i}-r_{i} Z_{i} Z_{1}+b Z_{i+1}+\mu K_{i_{0}, i} Z_{i_{0}} \\
\vdots \\
r_{i_{0}-2} Z_{i_{0}-2} Z_{1}-b Z_{i_{0}-1}-r_{i_{0}-1} Z_{i_{0}-1} Z_{1} \\
r_{i_{0}-1} Z_{i_{0}-1} Z_{1}-\left(\gamma+\frac{\mu}{2}\right) Z_{i_{0}}-\delta Z_{i_{0}} Z_{i_{0}+2 i_{f}} \\
\gamma Z_{i_{0}} \\
r_{f, 1} Z_{1}^{2}-b_{f} Z_{2}-r_{f, 2} Z_{i_{0}+2} Z_{1}+b_{f} Z_{i_{0}+3}-\gamma_{f} Z_{i_{0}+2} \\
\vdots \\
r_{f, j-1} Z_{i_{0}+j-1} Z_{1}-b_{f} Z_{i_{0}+j}-r_{f, j} Z_{i_{0}+j} Z_{1}+b_{f} Z_{i_{0}+j+1}-\gamma_{f} Z_{i_{0}+j} \\
\vdots \\
r_{f, i_{f}-1} Z_{i_{0}+i_{f}-2} Z_{1}-b_{f} Z_{i_{0}+i_{f}}-\gamma_{f} Z_{i_{0}+i_{f}} \\
-b_{p} Z_{i_{0}+i_{f}+1}+b_{p} Z_{i_{0}+i_{f}+2}+\gamma_{f} Z_{i_{0}+2} \\
\vdots \\
-b_{p} Z_{i_{0}+i_{f}+j-1}+b_{p} Z_{i_{0}+i_{f}+j}+\gamma_{f} Z_{i_{0}+j} \\
\vdots \\
-b_{p} Z_{i_{0}+2 i_{f}-1}+\gamma_{f} Z_{i_{0}+1_{f}} \\
-\delta Z_{i_{0}} Z_{i_{0}+2 i_{f}}-\alpha Z_{i_{0}+2 i_{f}} \\
\alpha Z_{i_{0}+2 i_{f}} \\
\delta Z_{i_{0}} Z_{i_{0}+2 i_{f}}
\end{array}\right.
$$

where

$$
\begin{aligned}
H_{1,1}(Z)= & -2\left(r_{1} Z_{1}^{2}-c Z_{2}\right)-\sum_{i=2}^{i_{0}-2}\left(r_{i} Z_{i} Z_{1}-b Z_{i+1}\right)-r_{i_{0}-1} Z_{i_{0}-1} Z_{1} \\
& -2\left(r_{f, 1} Z_{1}^{2}-b_{f} Z_{i_{0}+2}\right)-\sum_{j=2}^{i_{f}-1}\left(r_{f, j} Z_{i_{0}+j}+Z_{1}-b Z_{i_{0}+j+1}\right) \\
& +2 b_{p} Z_{i_{0}+i_{f}+1}+\sum_{j=2}^{i_{f}-1} b_{p} Z_{i_{0}+i_{f}+j} .
\end{aligned}
$$

We can easily see that $H_{1}$ is a locally Lipschitz continuous function with respect to $Z$. The Cauchy-Lipschitz theorem gives local existence and uniqueness of solutions for the system on $[0, \tilde{\tau}]$, for $\tilde{\tau}$ less than or equal to $\tau$. The global existence of solution on $[0, \tau)$ requires the solution to be bounded and non-negative on $[0, \tau)$. One can easily verifies that $H_{1}$ is bounded on $[0, \tau)$. 
To prove the non-negativity of the solution, we use the fact that an ODE system $y^{\prime}=f(y)$ on $\mathbb{R}^{n}$ is called quasi-positive if the condition

$$
y_{i} \geqslant 0, i \neq k \text { and } y_{k}=0 \Longrightarrow f_{k}(y) \geqslant 0
$$

is valid for all $k$ in $\{1, \cdots, n\}$ (Prüss et al., 2006). In our case, the system of equations (1)-(9) is quasi-positive (for $i$ in $\left\{2, \cdots, i_{0}-2\right\}$ and $j$ in $\left\{2, \cdots, i_{f}-1\right\}$ ):

$$
\begin{array}{lll}
c_{1}(t)=0 & \Longrightarrow c_{1}^{\prime}(t)=b c_{2}(t)+b \sum_{i=2}^{i_{0}-1} c_{i}(t)+b_{f} c_{f, 2}(t)+b_{p} c_{p l, 2}(t) & \\
& +\sum_{j=2}^{i_{f}}\left(b_{f} c_{f, j}(t)+b_{p} c_{p l, j}(t)\right) & \geqslant 0, \\
c_{i}(t)=0 & \Longrightarrow c_{i}^{\prime}(t)=r_{i-1} c_{i-1}(t) c_{1}(t)+b c_{i+1}(t)+\mu K_{i_{0}, i} c_{i_{0}}(t) & \geqslant 0, \\
c_{i_{0}-1}(t)=0 \Longrightarrow c_{i_{0}-1}^{\prime}(t)=r_{i_{0}-2} c_{i_{0}-2}(t) c_{1}(t) & \geqslant 0, \\
c_{i_{0}}(t)=0 & \Longrightarrow c_{i_{0}}^{\prime}(t)=r_{i_{0}-1} c_{i_{0}-1}(t) c_{1}(t) & \geqslant 0, \\
c_{p l}(t)=0 & \Longrightarrow c_{p l}^{\prime}(t)=\gamma c_{i_{0}}(t) & \geqslant 0, \\
c_{f, j}(t)=0 & \Longrightarrow c_{f, j}^{\prime}(t)=r_{f, j-1} c_{f, j-1}(t) c_{1}(t)+b_{f} c_{f, j+1}(t) & \geqslant 0, \\
c_{f, i_{f}}(t)=0 \Longrightarrow c_{f, i_{f}}^{\prime}(t)=r_{f, i_{f}-1} c_{f, i_{f}-1}(t) c_{1}(t) & \geqslant 0, \\
c_{p l, j}(t)=0 & \Longrightarrow c_{p l, j}^{\prime}(t)=b_{p} c_{p l, j+1}(t)+\gamma_{f} c_{f, j}(t) & \geqslant 0 . \\
c_{p l, i_{f}}(t)=0 \Longrightarrow c_{p l, i_{f}}^{\prime}(t)=\gamma_{f} c_{f, i_{f}}(t) &
\end{array}
$$

Moreover, non-negativity of solutions of equations (12)-(14) has been proven in (Ciuperca et al.). Solutions on $[0, \tilde{\tau})$ are bounded and non-negative on $[0, \tau)$ and are therefore global solutions on $[0, \tau)$, and we further define $Z(\tau)=\lim _{t \rightarrow \tau^{-}} Z(t)$.

On $[\tau, 2 \tau)$, the system of equations (1)-(14) can be written as $Z^{\prime}(t)=H_{2}(t, Z(t), Z(t-\tau))$, with initial conditions $Z(\tau)$, and where $H_{2}$ is a polynomial function. Because $Z(t-\tau)$ is known from the previous step, $H_{2}$ can be written as a function of $Z(t)$ only. Once again, it is a locally Lipschitz continuous function, bounded and non-negative on $[\tau, 2 \tau)$. Therefore, the CauchyLipschitz theorem gives existence and uniqueness of solutions on $[\tau, 2 \tau)$.

We finally extend this result on intervals $[n \tau,(n+1) \tau)$, for all $n$ in $\mathbb{N}$, and prove existence, uniqueness and non-negativity of solution on $[0,+\infty)$.

\subsection{Steady state}

In this section we present our main result, concerning the steady state of the model.

Proposition 2 System of equations (1)-(14) has a unique steady state, where all variables are equal to zero, except for $c_{p l}$ and $p_{o l}$ :

$$
\begin{aligned}
\bar{Z} & =\left[\bar{c}_{1}, \bar{c}_{2}, \cdots, \bar{c}_{i_{0}}, \bar{c}_{p l}, \bar{c}_{f, 2}, \cdots, \bar{c}_{f, i_{f}}, \bar{c}_{p l, 2}, \cdots, \bar{c}_{p l, i_{f}}, \bar{p}_{c}, \bar{p}_{o l}, \bar{X}\right], \\
& =\left[0,0, \cdots, 0, \frac{Q}{i_{0}}, 0, \cdots, 0,0, \cdots, 0, \frac{Q_{p}}{m_{p}}, 0,0\right] .
\end{aligned}
$$

Besides, this steady state is globally stable.

Proof We first define a reduced system, using mass equations (16)-(17) and properties of equation (14). Indeed, we can express $c_{p l}$ and $p_{o l}$ as function of masses and other variables:

$$
\begin{aligned}
& \left.c_{p l}=\frac{1}{i_{0}}\left(Q-c_{1}(t)-\sum_{i=2}^{i_{0}} i c_{i}(t)-\sum_{j=2}^{i_{f}} j\left(c_{f, j}(t)+c_{p l, j}(t)\right)-i_{0} X(t)\right)\right), \\
& p_{o l}(t)=\frac{Q_{p}}{m_{p}}-\left(p_{c}(t)+X(t)\right) .
\end{aligned}
$$


Equation (14) can be written as:

$$
X(t)=\delta \int_{t-\tau}^{t} c_{i_{0}}(s) p_{c}(s) d s,
$$

for $t$ in $[\tau,+\infty)$. This formulation is obtained by integrating equation (14) on $[\tau,+\infty)$ and by replacing $X(\tau)$ by its value obtained when we integrate equation (14) on $[0, \tau)$.

We finally focus on the system of equations (1)-(14), in which we remove equations (5), (13) and (14). This reduced system has a unique trivial steady state. Equations (20)-(22) give us the steady state for the whole system.

Steady state global stability is obtained through the following Lyapunov function:

$$
\begin{aligned}
V(Z)= & \sum_{i=1}^{i_{0}-1} i c_{i}(t)+\sum_{j=2}^{i_{f}} j\left(c_{f, j}(t)+c_{p l, j}(t)\right)+\left(i_{0}-1\right) c_{i_{0}}(t)+p_{c}(t)+X(t) \\
& +\mu \sum_{i=2}^{i_{0}-2} i \frac{K_{i_{0}, i}}{\gamma}\left(\frac{Q}{i_{0}}-c_{p l}(t)\right)+\left(i_{0}-1\right)\left(\frac{Q_{p}}{m_{p}}-p_{o l}(t)\right) .
\end{aligned}
$$

A sketch of its construction is given below.

We define the general form of the Lyapunov function:

$$
V(Z)=\sum_{j=1}^{j_{N}} D_{j}\left(\bar{Z}_{j}-Z_{j}\right)+\sum_{j=j_{N}+1}^{N} D_{j} Z_{j}
$$

where $N$ is the number of functions, $D_{j}$ are positive coefficients to determine and $\bar{Z}$ is the steady state we study. In this form, $Z_{j}$ with $j$ in $\left\{1, \cdots, j_{N}\right\}$ corresponds to variables with a non-trivial steady state. In our case, these functions are thus $c_{p l}$ and $p_{o l}$.

By construction, this Lyapunov function is equal to zero only at steady state and is positive elsewhere. Indeed, we can note that each variable is upper-bounded, using mass equations (16)(17). Specifically, $c_{p l}$ is upper-bounded by $\frac{Q}{i_{0}}$ and $p_{o l}$ by $\frac{Q_{p}}{m_{p}}$, corresponding to the values of $\bar{c}_{p l}$ and $\bar{p}_{\text {ol }}$.

We now have to choose coefficients $D_{j}$ so that $V^{\prime}$ is non-positive. Let us write the $V$ expression in our case:

$$
\begin{aligned}
V & =\underbrace{\sum_{i=1}^{i_{0}-1} S_{i} c_{i}(t)+\sum_{j=1}^{i_{f}} M_{j} c_{f, j}(t)+\sum_{j=1}^{i_{f}} N_{j} c_{p l, j}(t)}_{V_{1}} \\
& +\underbrace{D_{1}\left(\frac{Q}{i_{0}}-c_{p l}(t)\right)+D_{2}\left(\frac{Q_{p}}{m_{p}}-p_{o l}(t)\right)+D_{3} X(t)+D_{4} p_{c}(t)+D_{5} c_{i_{0}}(t)}_{V_{2}}
\end{aligned}
$$

where $S_{i}, i$ in $\left\{1, \cdots, i_{0}-1\right\}, M_{j}, N_{j}, j$ in $\left\{2, \cdots, i_{f}\right\}$, and $D_{k}, k$ in $\{1, \cdots, 5\}$ are positive coefficients to determine, to obtain non-positive derivative of $V$.

Taking the $V_{1}$ derivative, according to equations (1)-(9) gives us:

$$
V_{1}^{\prime}=W_{1}+W_{2}+W_{3},
$$


where $W_{1}$ corresponds to the oligomerization process part, $W_{2}$ to the fibrillation part and $W_{3}$ to the fibrillation in plaque part. $W_{1}$ can be written as follows:

$$
\begin{aligned}
W_{1} & =\sum_{i=2}^{i_{0}-1} S_{i}\left(G_{i-1}(t)-G_{i}(t)+\mu K_{i_{0}, i} c_{i_{0}}(t)\right)+S_{i_{0}-1} G_{i_{0}-2}(t)-S_{i_{0}-1} r_{i_{0}-1} c_{i_{0}-1}(t) c_{1}(t) \\
& -2 S_{1} G_{1}(t)-S_{1} \sum_{i=2}^{i_{0}-2} G_{i}(t)-S_{1} r_{i_{0}-1} c_{i_{0}-1}(t) c_{1}(t)
\end{aligned}
$$

where $G_{i}(t)=r_{i} c_{i}(t) c_{1}(t)-b c_{i+1}(t), i \in\left\{1, \cdots, i_{0}-2\right\}$ and $G_{i_{0}-1}(t)=r_{i_{0}-1} c_{i_{0}-1}(t) c_{1}(t)$. With these notations, we obtain:

$$
W_{1}=-r_{i_{0}-1} c_{i_{0}-1}(t) c_{1}(t)\left(S_{i_{0}-1}+S_{1}\right)+\sum_{i=2}^{i_{0}-1} S_{i} \mu K_{i_{0}, i} c_{i_{0}}(t)+\sum_{i=1}^{i_{0}-2} G_{i}(t)\left(S_{i+1}-S_{i}-S_{1}\right) .
$$

We take $S_{i}=i S_{1}$, for $i$ in $\left\{2, \cdots i_{0}-1\right\}$.

$W_{2}$ can be written as follows (denoting $M_{1}=S_{1}$ ):

$$
\begin{aligned}
W_{2} & =\sum_{j=2}^{i_{f}-1} M_{j}\left(F_{j-1}(t)-F_{j}(t)-\gamma_{f} c_{f, j}(t)\right)+M_{i_{f}} F_{i_{f}-1}(t)-M_{i_{f}} \gamma_{f} c_{f, i_{f}}(t) \\
& -2 S_{1} F_{1}(t)-S_{1} \sum_{j=2}^{i_{f}-1} F_{j}(t),
\end{aligned}
$$

where $F_{1}(t)=r_{f, 1} c_{1}(t)^{2}-b_{f} c_{f, 2}(t)$ and $F_{i}(t)=r_{f, i} c_{f, i}(t) c_{1}(t)-b_{f} c_{f, i+1}(t), i \in\left\{2, \cdots, i_{f}-1\right\}$. We then obtain:

$$
W_{2}=-\gamma_{f} \sum_{j=2}^{i_{f}} M_{j} c_{f, j}(t)+\sum_{j=1}^{i_{f}-1} F_{j}(t)\left(M_{j+1}-M_{j}-S_{1}\right) .
$$

In this case, we take $M_{j}=j S_{1}$, for $j$ in $\left\{2, \cdots i_{f}\right\}$.

$W_{3}$ can be written as follows, denoting $N_{1}=S_{1}$ and with $P_{i}(t)=-b_{p} c_{p, i+1}(t), i \in\left\{1, \cdots, i_{f}-1\right\}$ :

$$
\begin{aligned}
W_{3} & =\sum_{j=2}^{i_{f}-1} N_{j}\left(P_{j-1}(t)-P_{j}(t)+\gamma_{f} c_{f, j}(t)\right)+N_{i_{f}} P_{i_{f}-1}(t)+N_{i_{f}} \gamma_{f} c_{f, i_{f}}(t) \\
& -2 S_{1} P_{1}(t)-S_{1} \sum_{j=2}^{i_{f}-1} P_{j}(t) \\
W_{3} & =\gamma_{f} \sum_{j=2}^{i_{f}} N_{j} c_{f, j}(t)+\sum_{j=1}^{i_{f}-1} P_{j}(t)\left(N_{j+1}-N_{j}-S_{1}\right) .
\end{aligned}
$$

Once again, we take $N_{j}=j S_{1}$, for $j$ in $\left\{2, \cdots i_{f}\right\}$.

Finally $V_{1}^{\prime}$ is:

$$
V_{1}^{\prime}=-r_{i_{0}-1} c_{i_{0}-1}(t) c_{1}(t)\left(S_{i_{0}-1}+S_{1}\right)+\sum_{i=2}^{i_{0}-1} S_{i} \mu K_{i_{0}, i} c_{i_{0}}(t)
$$


The derivative of $V_{2}$ has the following expression:

$$
\begin{aligned}
V_{2}^{\prime} & =\delta c_{i_{0}}(t) p_{c}(t)\left(D_{3}-D_{4}-D_{5}\right)+\delta c_{i_{0}}(t-\tau) p_{c}(t-\tau)\left(D_{5}-D_{2}-D_{3}\right) \\
& +D_{5} r_{i_{0}-1} c_{i_{0}-1}(t) c_{1}(t)-c_{i_{0}}(t)\left(D_{5}\left(\gamma+\frac{\mu}{2}\right)+\gamma D_{1}\right)-\alpha p_{c}(t)\left(D_{2}+D_{4}\right) .
\end{aligned}
$$

We choose $D_{3}=D_{4}$ and $D_{2}=D_{5}$ to ensure negativity of the two first terms.

We finally have the following expression for $V^{\prime}$, with the different choices made for coefficient values:

$$
\begin{aligned}
V^{\prime} & =-r_{i_{0}-1} c_{i_{0}-1}(t) c_{1}(t)\left(i_{0} S_{1}-D_{5}\right)+c_{i_{0}}(t)\left(S_{1} \mu \sum_{i=2}^{i_{0}-1} i K_{i_{0}, i}-D_{5}\left(\gamma+\frac{\mu}{2}\right)-D_{1} \gamma\right) \\
& -D_{5} \delta c_{i_{0}}(t) p_{c}(t)-D_{3} \delta c_{i_{0}}(t-\tau) p_{c}(t-\tau)-\alpha p_{c}(t)\left(D_{2}+D_{4}\right) .
\end{aligned}
$$

Let us recall that this derivative should be equal to zero at steady state and negative elsewhere. To guarantee this, we finally choose $D_{5}=\left(i_{0}-1\right) S_{1}$ and $D_{1}=\frac{\mu \sum_{i=2}^{i_{0}-1} i K_{i_{0}, i}}{\gamma} S_{1}$. Expression (23) is finally obtained by taking $S_{1}=1$. By construction this function is equal to zero at steady state and positive elsewhere, with a non-positive derivative. One can easily verify that the derivative is equal to zero only at steady state.

We prove that this model admits a unique solution, which remains non-negative provided that the initial conditions are non-negative. Moreover, we find a unique steady state, which is globally stable. From a biological point a view, this means that regardless of the initial conditions of the experiment, all monomers will eventually polymerize into oligomers that will be transported in the amyloid plaque, while all $\mathrm{PrP}^{\mathrm{C}}$ prions will be misfolded in $\mathrm{PrP}^{\mathrm{ol}}$ prions.

In the following section, we focus on the global kinetics, to comprehend the role and the impact of each parameter on the dynamics without any experimental data.

\section{Numerical analysis}

We perform a sensitivity analysis and different numerical simulations. We focus on the impact of model parameters on the dynamics of prion proteins, as they might drive the neuronal loss in Alzheimer's disease, and is the new feature in this model.

\section{Numerical simulations}

First, we randomly sample 5000 parameter sets and simulate the model with each of them. We arbitrarily set $i_{0}=20$ and $i_{f}=40$, and choose $r_{i}=r_{f, i}=a \times i$ as the polymerization rate for proto-oligomers and fibrils, where $i$ is the polymer size (number of monomers it contains). Initial values for monomers and prions are respectively $m=1$ and $p=1$. We keep a set of parameters for which all $\mathrm{PrP}^{\mathrm{C}}$ are misconformed in $\mathrm{PrP}^{\mathrm{ol}}$. To prevent a direct transformation of $\mathrm{PrP}^{\mathrm{C}}$ in $\mathrm{PrP}^{\mathrm{ol}}$, we force $\alpha$ to take a small value. Parameter values are the following, and the corresponding simulations are displayed on Figure 2. Note that these parameters should next be estimated with experimental data, to determine optimal values and corresponding units. However, to the best of our knowledge such data are still really challenging to obtain.

$$
\begin{array}{lll}
i_{0}=20, & i_{f}=40, & a=4.7113, \\
b=3.0945, & b_{f}=2.3194, & b_{p}=3.6038, \\
\gamma=0.0358, & \gamma_{f}=0.9675, & \delta=3.7945, \\
\tau=9.4082, & \mu=0.0168, & \alpha=0.001 .
\end{array}
$$



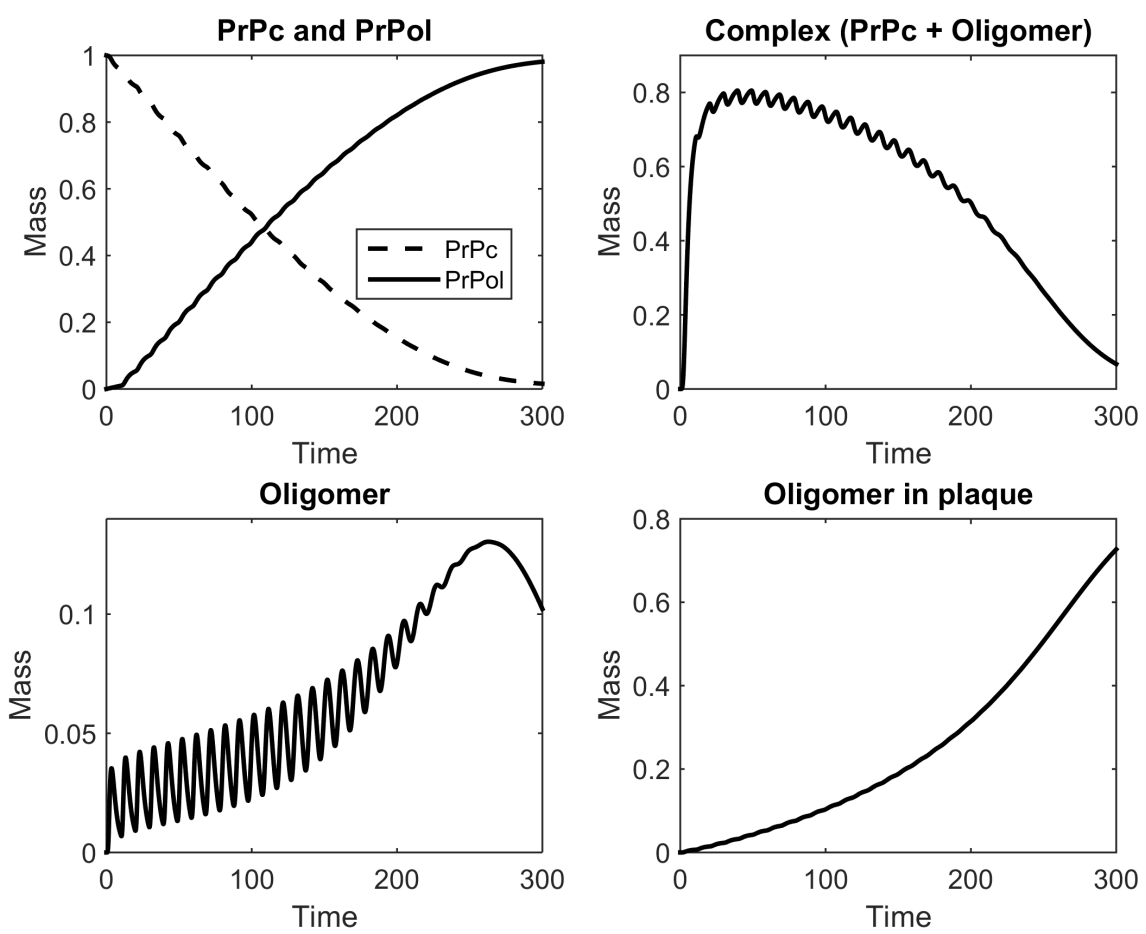

Fig. 2: Numerical simulations with parameter values as given in the main text. Top left: Time evolution of prions $\mathrm{PrP}^{\mathrm{C}}$ (dashed line) and oligomeric prions $\mathrm{PrP}^{\circ}$ (solid line). Top right: Time evolution of $\mathrm{A} \beta / \mathrm{PrP}{ }^{\mathrm{C}}$ complex mass (assumed to be $i_{0}$ ). Bottom left: Time evolution of oligomer mass. Bottom right: Time evolution of the mass of oligomers in plaque.

As expected, the mass of oligomeric prions tends to the $\mathrm{PrP}^{\mathrm{C}}$ initial value, as it is the steady state value (see Section 3.2). Similarly, the mass of oligomers in the plaque tends to the initial mass of $\mathrm{A} \beta$ monomers. The misconformation of $\mathrm{PrP}^{\mathrm{C}}$ in $\mathrm{PrP}^{\mathrm{ol}}$ is mainly due to the interaction between prions and oligomers, as the value of direct transformation rate $\alpha$ is small. Because the reaction rate between $\mathrm{PrP}^{\mathrm{C}}$ and $\mathrm{A} \beta$ oligomers is greater than the displacement rate of oligomers into the plaque $(\delta>\gamma)$, oligomers are more likely to interact with prions to form complexes. After $\tau$ units of time, they are released from this complex, causing oscillations in the evolution of complexes and oligomers. The formation of complexes decreases with time as less $\mathrm{PrP}^{\mathrm{C}}$ are remaining.

\section{Sensitivity analysis}

To better understand the impact of each parameter on the emergence of $\mathrm{PrP}^{\mathrm{ol}}$, we perform a sensitivity analysis through first order Sobol' indices (Iooss and Lemaittre, 2015) given by the following expression:

$$
S_{i}=\frac{V\left(\mathbf{E}\left[Y \mid X_{i}\right]\right)}{V(Y)}, \quad i=1, \cdots N
$$

where $Y$ is the model output (evolution of $\mathrm{PrP}^{\text {ol }}$ in our case), $X_{i}$ is a model parameter, $V(Y)$ represents the total variance of $Y, \mathbf{E}\left[Y \mid X_{i}\right]$ is the conditional mean of $Y$ given $X_{i}$, and $N$ is the number of model parameters. First order Sobol' indices $S_{i}$ determine how much the model output varies when a parameter value varies. A parameter associated with a Sobol' index close to 
1 has a large impact on $Y$ variability, meaning that the model output is very sensitive to change in this parameter. In this work, we apply a sensitivity analysis to highlight the impact of each parameter on $\mathrm{PrP}^{\mathrm{ol}}$ emergence and to determine which ones contribute most to the output. We limit our study to first order Sobol' indices. Note that the impact of the interaction of several parameters on model output can be also be assessed through Sobol' indices, but can be more difficult to interpret. Because we do not know ranges of values for the different parameters, the interpretation of Sobol' indices should be taken with caution. Figure 3 displays results of sensitivity analysis: for each parameter, Sobol' index is calculated and its evolution with time is represented.

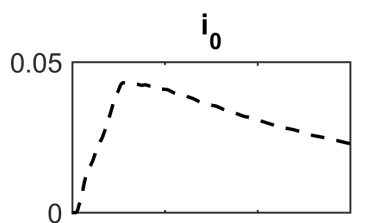

b

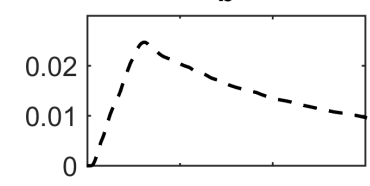

$\gamma$

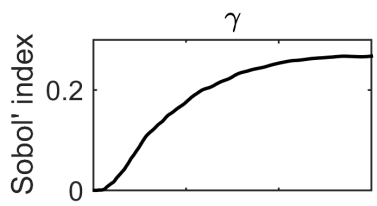

$\tau$

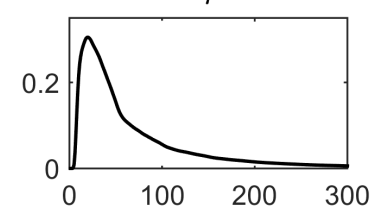

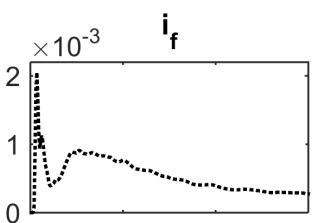
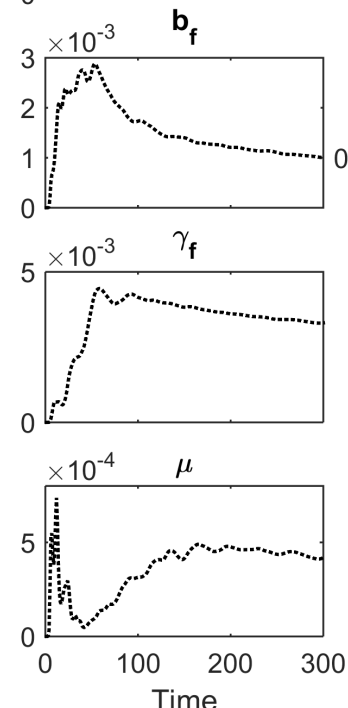

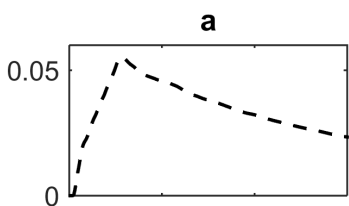

$\mathbf{b}_{\mathrm{p}}$

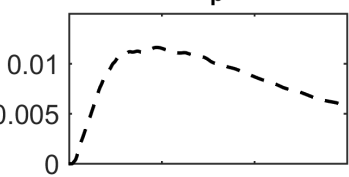

$\delta$

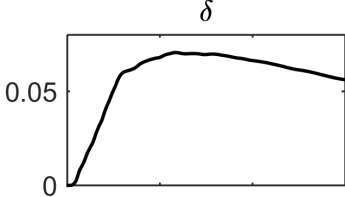

$\alpha$

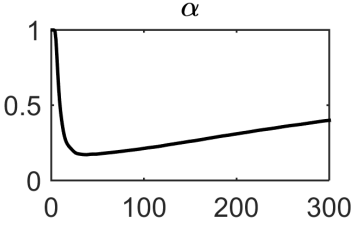

Fig. 3: Evolution of Sobol' indices with time for each parameter. Solid lines are used for parameters impacting $\mathrm{PrP}^{\mathrm{ol}}$ emergence most, dashed lines are used for parameters with a small impact and dotted lines for parameters with no impact.

Based on these results, parameters can be divided in three groups:

- parameters that impact model output most (solid lines on Figure 3): displacement rate of oligomers in plaque $\gamma$, reaction rate between $\mathrm{A} \beta$ oligomers and $\operatorname{PrP}^{\mathrm{C}} \delta$, duration of oligomers and $\operatorname{PrP}^{\mathrm{C}}$ interaction $\tau$ and rate of direct transformation of $\operatorname{PrP}^{\mathrm{C}}$ into $\operatorname{PrP}^{\mathrm{ol}} \alpha$,

- parameters having little impact on model output (dashed lines on Figure 3): oligomer size $i_{0}$, polymerization rate $a\left(r_{i}=r_{f, i}=a \times i\right)$ and depolymerization rates of proto-oligomers $b$ and fibrils in plaque $b_{p}$, 
- parameters having no impact on model output (dotted lines on Figure 3): maximal fibril size $i_{f}$, depolymerization rate of fibrils $b_{f}$, displacement rate of fibrils in plaque $\gamma_{f}$ and proportion of oligomer fragmentation $\mu$.

This is consistent with the role of each parameter. Indeed, we expect that parameters related to $\mathrm{A} \beta$ oligomers $/ \operatorname{PrP}^{\mathrm{C}}$ interaction play a greater role in the emergence of $\operatorname{PrP}^{\mathrm{ol}}$. As $\mathrm{A} \beta$ oligomers are required to misconform $\mathrm{PrP}^{\mathrm{C}}$ in $\mathrm{PrP}^{\text {ol }}$, it seems reasonable that parameters related to oligomer formation also impact $\mathrm{PrP}^{\mathrm{ol}}$ emergence. However, fibrils do not interact with $\mathrm{PrP}^{\mathrm{C}}$ and therefore do not impact $\mathrm{PrP}^{\mathrm{ol}}$ evolution. To go further, we illustrate the impact of parameters a (polymerization rate), $b$ (proto-oligomer depolimerization rate), $\gamma$ (diplacement rate of oligomers in plaque) and $\delta$ (reaction rate between $\mathrm{A} \beta$ oligomers and $\mathrm{PrP}^{\mathrm{C}}$ ). These parameters are chosen as they have a relatively strong impact on $\mathrm{PrP}^{\mathrm{ol}}$ evolution. Moreover, the processes they describe could constitute new therapeutic targets to slow down the emergence of $\mathrm{PrP}^{\mathrm{ol}}$, and thus the progression of Alzheimer's disease.

\section{Impact of displacement rate of oligomers into the plaque $\gamma$}

Figure 4 displays the impact of parameter $\gamma$, the displacement rate of $\mathrm{A} \beta$ oligomers into plaque, on the evolution of $\mathrm{PrP}^{\mathrm{ol}}$. We perform model simulations with different values for this parameter while the others are fixed to the values given previously. We represent the evolution of $\mathrm{PrP}^{\mathrm{ol}}(\mathrm{Fig}-$ ure 4, top left) along with evolutions of $\mathrm{A} \beta / \mathrm{PrP}^{\mathrm{C}}$ complexes (top right), $\mathrm{A} \beta$ oligomers (bottom left) and oligomers in plaque (bottom right). These simulations represent the impact of $\gamma$ on the dynamics described by the model. As expected, for small values of $\gamma, \mathrm{A} \beta$ oligomers are less likely to be transported to the plaque, and therefore can bind to $\operatorname{PrP}^{\mathrm{C}}$. Consequently, more complexes are created, leading to a faster emergence of $\mathrm{PrP}^{\mathrm{ol}}$. On the contrary, for larger values of $\gamma$, the mass of $\mathrm{A} \beta$ oligomers in plaque increases faster, and therefore less $\mathrm{PrP}^{\mathrm{C}}$ are misconformed in $\mathrm{PrP}^{\mathrm{ol}}$.

\section{Impact of reaction rate between oligomers and $\operatorname{PrP}^{\mathrm{C}} \delta$}

Figure 5 displays the impact of $\delta$, the reaction rate between $\mathrm{A} \beta$ oligomers and prions $\operatorname{PrP}^{\mathrm{C}}$ on evolutions of $\mathrm{PrP}^{\mathrm{ol}}$ (top left), complexes (top right), oligomers (bottom left) and oligomers in plaque (bottom right). For small values of $\delta, \mathrm{A} \beta$ oligomers are less likely to interact with $\mathrm{PrP}^{\mathrm{C}}$ than to be transported in the plaque. This is the reason why we observe a stronger increase of the mass of oligomers in the plaque, while few complexes are created. For greater values of $\delta, \mathrm{A} \beta$ oligomers interact with $\mathrm{PrP}^{\mathrm{C}}$ and therefore more $\mathrm{PrP}^{\mathrm{ol}}$ are created. We observe that for these values oligomers are found in the form of complexes or in the plaque, but few are left free.

\section{Impact of polymerization rate $a$}

We also study the impact of polymerization parameters on the emergence of $\mathrm{PrP}^{\mathrm{ol}}$. Although the polymerization rate $a$ does not explicitly take part in the interaction sub-model, it impacts the evolution of $\mathrm{PrP}^{\mathrm{ol}}$ through the formation of oligomers. Results of simulations for this parameter are displayed on Figure 6. We observe that for small values of $a$, few $\mathrm{PrP}^{\mathrm{ol}}$ are created. Indeed, because the polymerization rate is small, few oligomers are formed and therefore the interaction between $\mathrm{PrP}^{\mathrm{C}}$ and $\mathrm{A} \beta$ oligomers is less likely to occur. As $a$ value increases, we observe an increase in the mass of complexes and of oligomers, leading to a faster emergence of $\mathrm{PrP}^{\mathrm{ol}}$. We notice that the decrease of $a$ value also slows down the increase of oligomer mass into the plaque. Indeed, as less oligomers are created, less are transported into the amyloid plaque. Consequently, the system will take more time to reach the steady state and $\mathrm{A} \beta$ monomers remain free in the brain. 

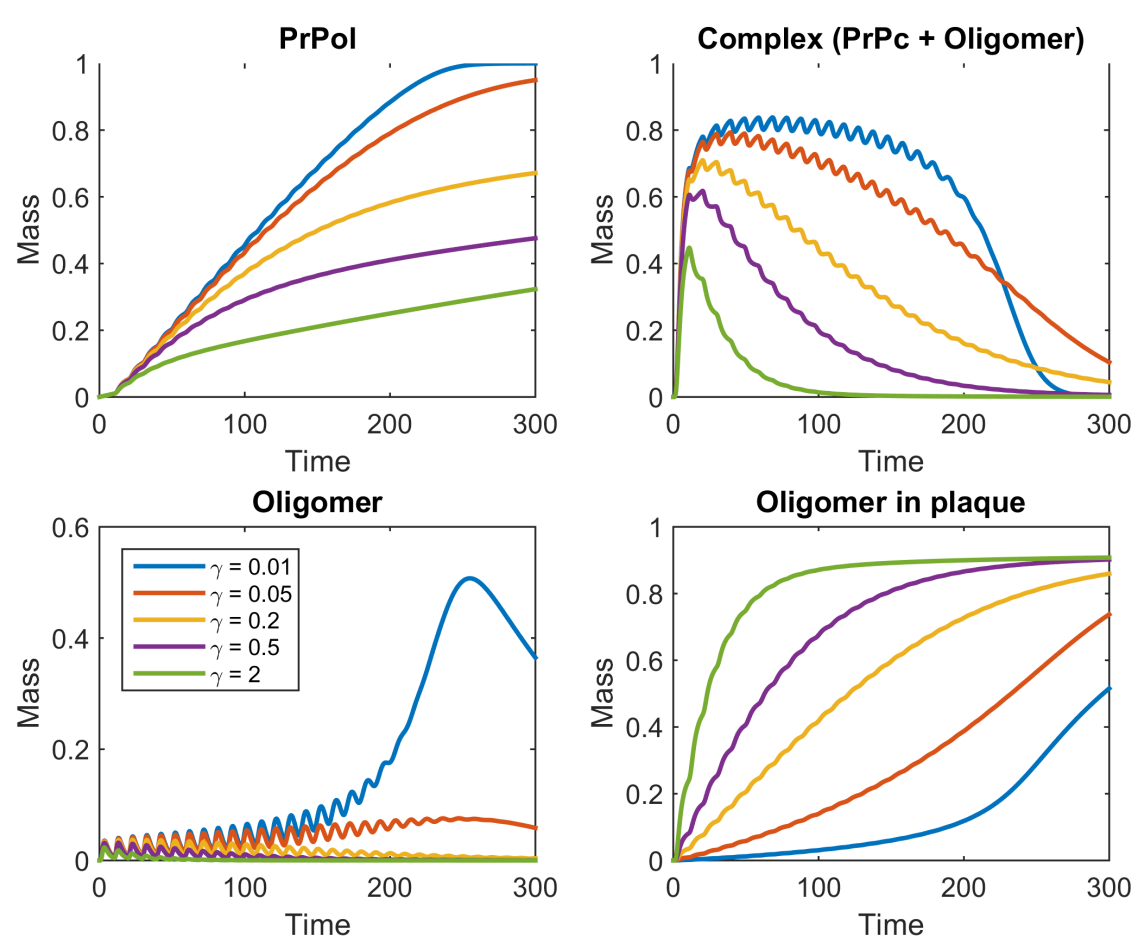

Fig. 4: Impact of the displacement rate of oligomers into plaque $\gamma$ on different outputs. Simulations are obtained with different values of $\gamma(\gamma=0.01$ : blue curve, $\gamma=0.05$ : red curve, $\gamma=0.2$ : yellow curve, $\gamma=0.5$ : purple curve and $\gamma=2$ : green curve), while other parameters are fixed to the values given previously. Top left: evolution of oligomeric prions $\mathrm{PrP}^{\mathrm{ol}}$. Top right: evolution of $\mathrm{A} \beta / \mathrm{PrP}^{\mathrm{C}}$ complexes. Bottom left: evolution of $\mathrm{A} \beta$ oligomers. Bottom right: evolution of $\mathrm{A} \beta$ oligomers in plaque.

\section{Impact of depolymerization rate $b$}

We finally present results of simulations for different values of the depolymerization rate of $\mathrm{A} \beta$ proto-oligomers $b$ (Figure 7). For this parameter, we observe two cases. For small values of $b$, proto-oligomers do not depolymerize a lot, and therefore the monomer reserve cannot renew. Consequently, proto-oligomers have difficulties to evolve in bigger structures and to form oligomers. Prions $\operatorname{PrP}^{\mathrm{C}}$ are then less likely to interact with $\mathrm{A} \beta$ oligomers and $\mathrm{PrP}^{\mathrm{ol}}$ emergence is slowed down. With greater values of $b, \operatorname{PrP}^{\mathrm{C}}$ are misconformed in $\mathrm{PrP}^{\mathrm{ol}}$ faster. Depolymerization of proto-oligomers is stronger and enables monomer concentration to remain at a sufficient level to induce polymerization process and formation of oligomers. This leads to the interaction between $\mathrm{A} \beta$ oligomers and $\operatorname{PrP}^{\mathrm{C}}$, and to the emergence of $\operatorname{PrP}^{\mathrm{ol}}$. Note that for $b=3$, less oligomers are present in the plaque at the end of the simulation, due to polymeriation slowing down. Similarly for the effect of parameter $a$, we observe that for small values of $b$, less oligomers are transported into the amyloid plaque.

\section{Plausible therapeutic approaches?}

These four parameters could constitute plausible new therapeutic targets to treat Alzheimer's disease, as they slow down the emergence of oligomeric prions, that are supposedly toxic for the neurons. However, they do not have the same impact on monomers and oligomers. Indeed, while 

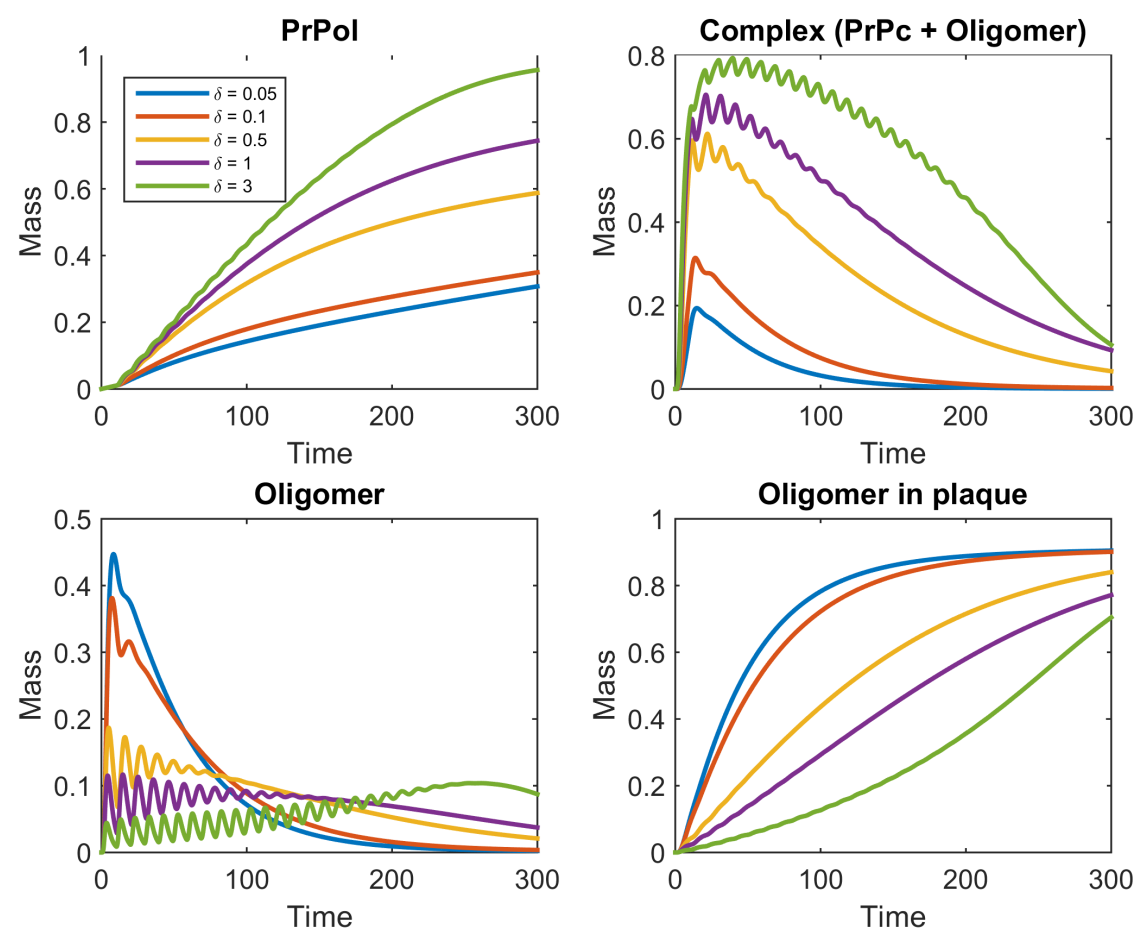

Fig. 5: Impact of the reaction rate between $\mathrm{A} \beta$ oligomers and $\operatorname{PrP}^{\mathrm{C}} \delta$ on different outputs. Simulations are obtained with different values of $\delta(\delta=0.05$ : blue curve, $\delta=0.1$ : red curve, $\delta=0.5$ : yellow curve, $\delta=1:$ purple curve and $\delta=3$ : green curve), while other parameters are fixed to the values given previously. Top left: evolution of oligomeric prions $\mathrm{PrP}^{\mathrm{ol}}$. Top right: evolution of $\mathrm{A} \beta / \mathrm{PrP}^{\mathrm{C}}$ complexes. Bottom left: evolution of $\mathrm{A} \beta$ oligomers. Bottom right: evolution of $\mathrm{A} \beta$ oligomers in plaque.

parameters $\gamma$ and $\delta$ favours one mechanism between $\mathrm{PrP}^{\mathrm{ol}}$ emergence and oligomer displacement into the plaque, parameters $a$ and $b$ also reduce the mass of oligomers in the plaque. It is then relevant to analyse monomer and proto-oligomer evolutions, to determine under which structures $\mathrm{A} \beta$ monomers could be found in the brain.

We perform four additional numerical simulations using parameter values as given previously. For each of these simulations, we replace $\gamma, \delta, a$ or $b$ values with the value that reduces the most the emergence of $\mathrm{PrP}^{\mathrm{ol}}$ (from previous simulations), and we compare the evolution of monomers, proto-oligomers of size 10, $\mathrm{PrP}^{\mathrm{ol}}$ and oligomers in the plaque (Figure 8). As expected, $\gamma$ and $\delta$ have the same impact on monomer and proto-oligomer evolutions, and similar results in terms of evolutions of $\mathrm{PrP}^{\mathrm{ol}}$ and oligomers in the plaque. However, because the value of $a$ is low, few protooligomers and oligomers are created and consequently monomers remain free in the brain. The effect of $b$ is quite the opposite: proto-oligomers are created, but because the depolymerization rate is low, the monomer reserve is not renewed and proto-oligomers cannot evolve in bigger structures and accumulate in the brain. 

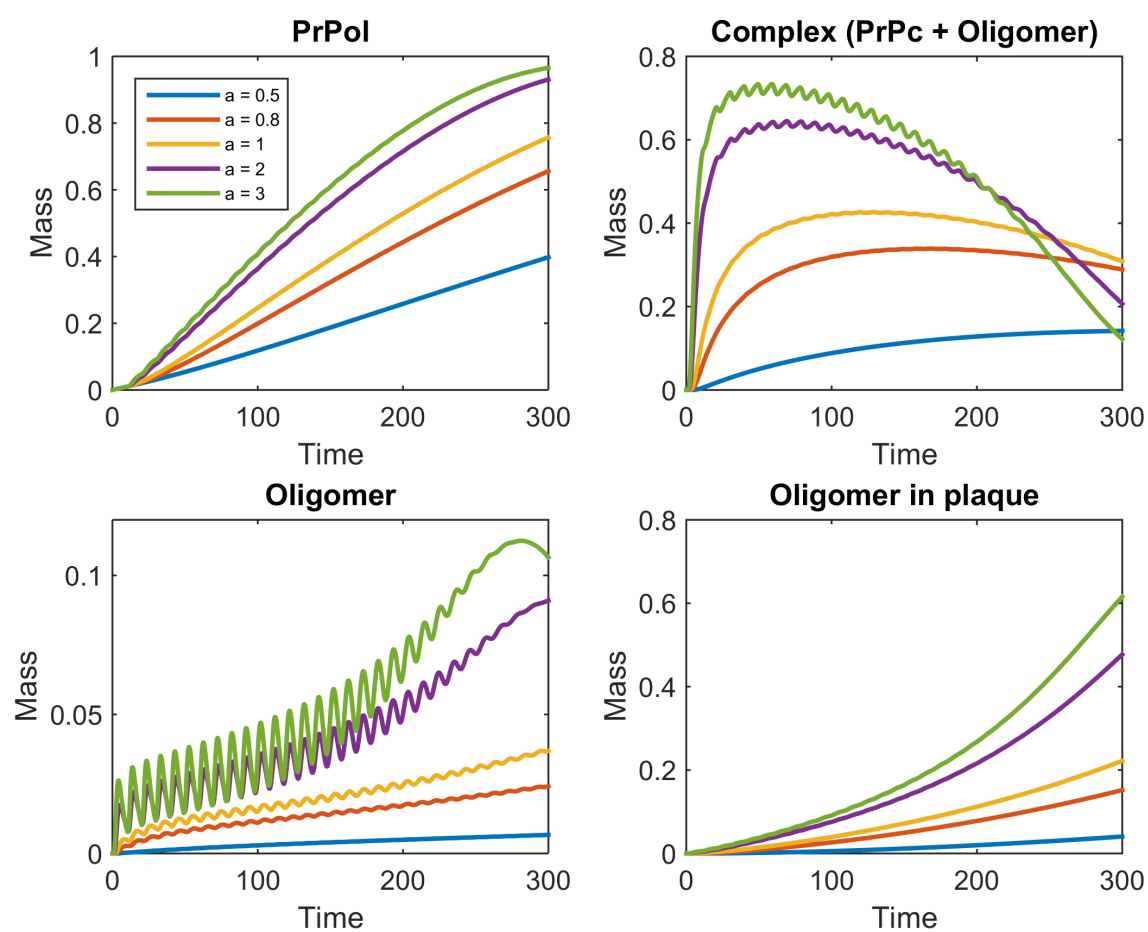

Fig. 6: Impact of the polymerization rate $a\left(r_{i}=r_{f, i}=a \times i\right)$ on different outputs. Simulations are obtained with different values of $a$ ( $a=0.5$ : blue curve, $a=0.8$ : red curve, $a=1$ : yellow curve, $a=2$ : purple curve and $a=3$ : green curve), while other parameters are fixed to the values given previously. Top left: evolution of oligomeric prions $\mathrm{PrP}^{\mathrm{ol}}$. Top right: evolution of $\mathrm{A} \beta / \mathrm{PrP}^{\mathrm{C}}$ complexes. Bottom left: evolution of $\mathrm{A} \beta$ oligomers. Bottom right: evolution of $\mathrm{A} \beta$ oligomers in plaque.

\section{Discussion}

$\mathrm{A} \beta$ oligomers and prions seem to play an important role in the emergence of Alzheimer's disease although it remains to be fully understood. Recent evidence suggests that oligomers can bind to neuron membrane receptors such as prions $\operatorname{PrP}^{\mathrm{C}}$. This interaction induces first a structural re-arrangement of $\mathrm{PrP}^{\mathrm{C}}$, leading then to its oligomerization into $\mathrm{PrP}^{\mathrm{ol}}$. This last phenomenon is responsible for the transmission of a death signal to neurons. This process has been proposed to be one of the mechanisms responsible of neuron lose Cissé and Mucke (2009); Laurén et al. (2009); Gimbel et al. (2010).

In this work, based on individually well characterized pathways, we present a mathematical model describing the formation of oligomers and their interaction with $\mathrm{PrP}^{\mathrm{C}}$. The sub-model for polymerization process (oligomerization and fibrillation) is composed of differential equations, based on Becker-Döring equations (Becker and Döring, 1935). They include different mechanisms: polymerization/depolymerization, displacement in amyloid plaque and fragmentation of oligomers. The sub-model for $\mathrm{A} \beta / \mathrm{PrP}^{\mathrm{C}}$ interaction is based on delayed differential equations and describes misfolding due to this interaction as well as direct transformation of $\mathrm{PrP}^{\mathrm{C}}$ into $\mathrm{PrP}^{\mathrm{ol}}$, due to possible other mechanisms we are not aware of. We prove that this model admits one 

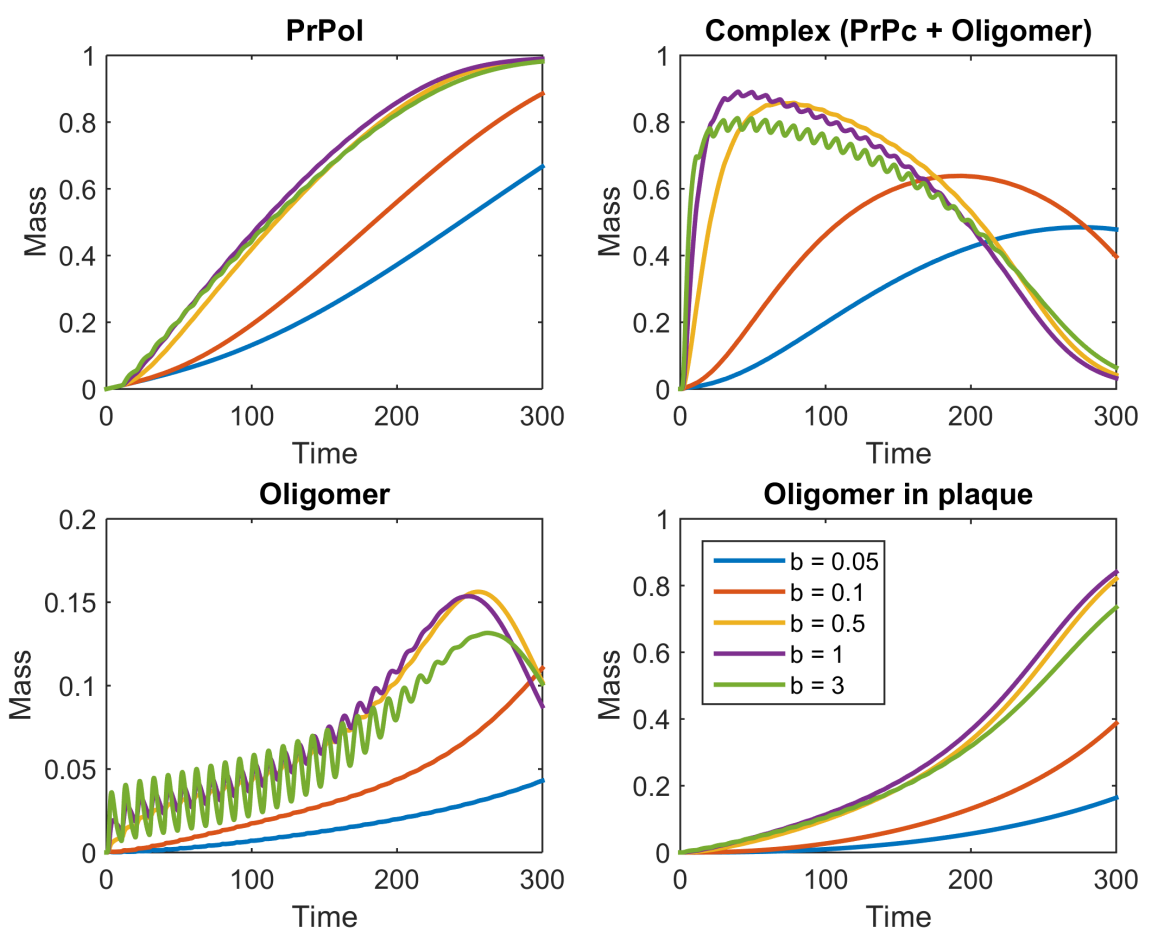

Fig. 7: Impact of the depolymerization rate of proto-oligomers $b$ on different outputs. Simulations are obtained with different values of $b(b=0.05$ : blue curve, $b=0.1$ : red curve, $b=0.5$ : yellow curve, $b=1$ : purple curve and $b=3$ : green curve), while other parameters are fixed to the values given previously. Top left: evolution of oligomeric prions $\operatorname{PrP}^{\mathrm{ol}}$. Top right: evolution of $\mathrm{A} \beta / \mathrm{PrP}^{\mathrm{C}}$ complexes. Bottom left: evolution of $\mathrm{A} \beta$ oligomers. Bottom right: evolution of $\mathrm{A} \beta$ oligomers in plaque.

unique solution, which remains non-negative for non-negative initial conditions. Moreover, the model has an unique non-trivial steady state. We demonstrate that this steady state is globally stable, through the construction of a Lyapunov function, which constitutes the main result of this work. Although the mathematical tools used in this work are quite standard, we believe that finding a rlevant Lyapunov function could prove to be quite challenging. From a biological point of view, this means that, regardless of the initial conditions, the system tends to a unique state, where all monomers have polymerized in oligomers and are in the amyloid plaques, while all prions are misconformed into oligomeric prions.

We analyse the model from a numerical point of view, using sensitivity analysis and numerical simulations. We highlight the impact of different parameters, namely the reaction rate between oligomers and $\operatorname{PrP}^{\mathrm{C}} \delta$, the displacement rate of oligomers in the plaque $\gamma$, the polymerization rate $a$ and the depolymerization rate of proto-oligomers $b$, on the evolution of $\mathrm{PrP}^{\mathrm{ol}}$. We show that small values of parameters $a$ and $b$ slow down the emergence of oligomeric prions, as they prevent a fast polymerization into oligomers. Moreover, as less oligomers are created, we see that the mass of oligomers in the amyloid plaque is drastically reduced, and therefore it will take more time for the system to reach the steady state. This is not observed for parameters $\delta$ and $\gamma$ because these parameters favour one mechanism between $\mathrm{A} \beta$ oligomers and $\mathrm{PrP}^{\mathrm{C}}$ interaction and 

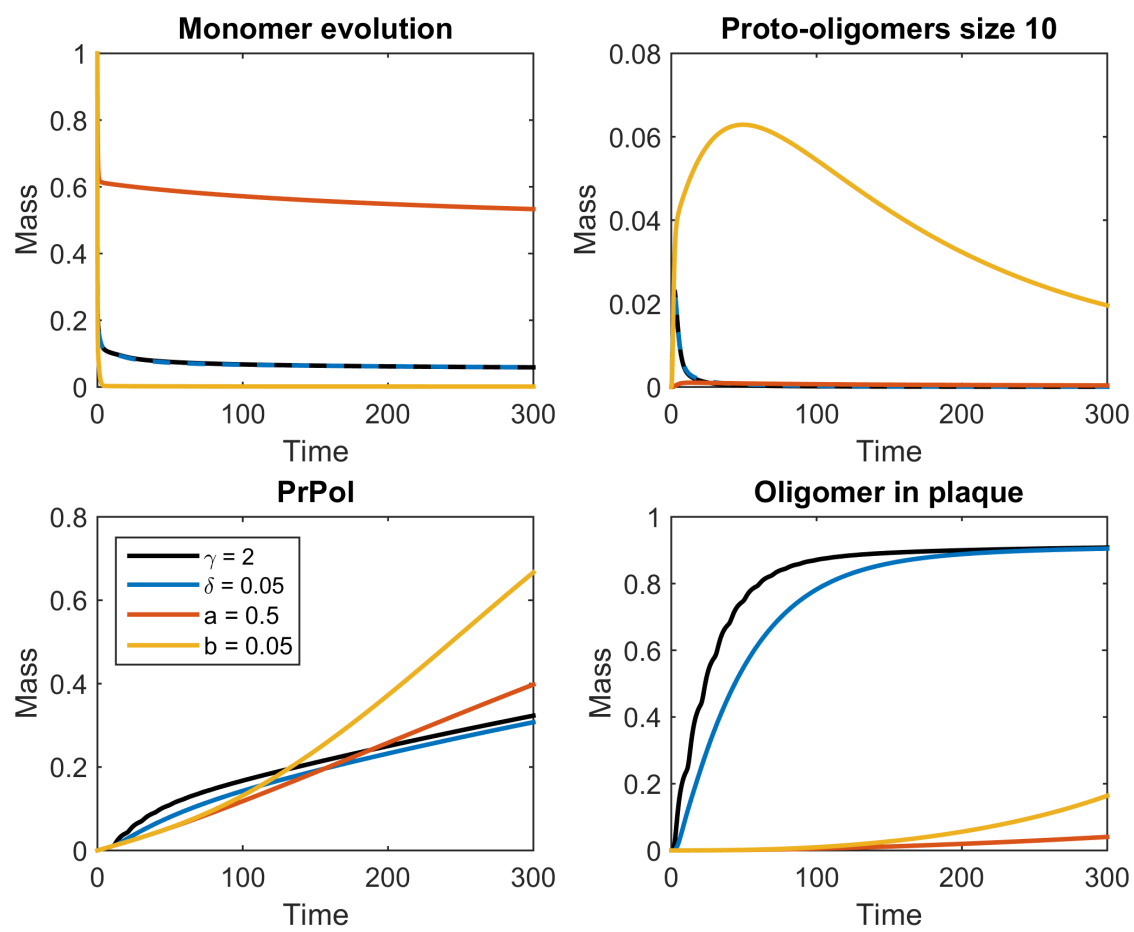

Fig. 8: Comparison between "best" values of $\gamma, \delta, a$ and $b$ parameters, $i e$ values that slow down the emergence of PrPol: $\gamma=2$ (black), $\delta=0.05$ (blue), $a=0.5$ (red) and $b=0.05$ (yellow). Top left: evolution of monomers. Top right: evolution of proto-oligomers of size $10\left(i_{0} / 2\right)$. Bottom left: evolution of oligomeric prions PrP ${ }^{\text {ol }}$. Bottom right: evolution of oligomers in the amyloid plaque.

oligomer displacement into the plaque. Although our results should be assessed with experimental data, we think that these processes could constitute targets for the pharmaceutical industry, to slow down the emergence of Alzheimer's disease (Canter et al., 2016). Different strategies could be considered. Reducing proto-oligomer polymerization or depolymerization may slow down the emergence of oligomers and therefore the emergence of oligomeric prions, but induces an accumulation of free monomers or proto-oligomers into the brain. Decreasing the reaction rate between oligomers and prions or increasing the transport of oligomers in the amyloid plaque may slow down the emergence of oligomeric prions, but oligomer mass in the plaque increases faster. One should then find the right balance between the emergence of oligomeric prions, the accumulation of monomers or proto-oligomers into the brain, and the increase of oligomers into the amyloid plaque.

To go further, experimental data is required to validate the model from a biological point of view, and estimate model parameters. Besides, the model should include two types of $\mathrm{A} \beta$ monomers: $\mathrm{A} \beta-40$, which is the most common type of monomers present in the brain, and $\mathrm{A} \beta-42$, whose presence may indicate the emergence of Alzheimer's disease. It could be interesting to study the impact of these two types of monomers on the whole dynamics. Indeed they do not have the same polymerization kinetics (Bitan et al., 2003; Johnson et al., 2013), and may not interact with 
prions in the same way, which could constitute another therapeutic strategy. However, without experimental data comparing their dynamics, it is quite difficult to model their differences.

Acknowledgements The authors thank Prof Glenn F. Webb for his valuable reading and corrections.

\section{References}

Y. Achdou, B. Franchi, N. Marcello, and M.C. Tesi. A qualitative model for aggregation and diffusion of $\beta$-amyloid in Alzheimer's disease. J. Math. Biol., 67(6-7):1369-92, 2013. ISSN 1432-1416.

B. Barz, Q. Liao, and B. Strodel. Pathways of amyloid- $\beta$ aggregation depend on oligomer shape. Journal of the American Chemical Society, 2017.

R. Becker and W. Döring. Kinetische Behandlung der Keimbildung in übersättigten Dämpfen. Ann. Phys., 24:719-752, 1935.

M. Bertsch, B. Franchi, N. Marcello, M.C. Tesi, and A. Tosin. Alzheimer's disease: a mathematical model for onset and progression. Math. Med. Biol., :, 2016.

G. Bitan, M.D. Kirkitadze, A. Lomakin, S.S. Vollers, G.B. Benedek, and D.B. Teplow. Amyloid $\beta$-protein $(\mathrm{A} \beta$ ) assembly: $\mathrm{A} \beta 40$ and $\mathrm{A} \beta 42$ oligomerize through distinct pathways. Proc. Natl. Acad. Sci. U. S. A., 100(1):330-335, 2003. ISSN 0027-8424.

V. Calvez, N. Lenuzza, D. Oelz, J-P. Deslys, P. Laurent, F. Mouthon, and B. Perthame. Size distribution dependence of prion aggregates infectivity. Math. Biosci., 217(1):88-99, 2009. ISSN 0025-5564.

R.G. Canter, J. Penney, and L-H. Tsai. The road to restoring neural circuits for the treatment of alzheimer's disease. Nature, 539(7628):187, 2016.

N. Carulla, G.L. Caddy, D.R. Hall, J. Zurdo, M. Gairí, M. Feliz, E. Giralt, C.V. Robinson, and C.M. Dobson. Molecular recycling within amyloid fibrils. Nature, 436(7050):554, 2005.

M. Cissé and L. Mucke. A prion protein connection. Nature, 457:1090-1091, 2009. ISSN 08866236.

I.S. Ciuperca, M. Dumont, A. Lakmeche, P. Mazzocco, L. Pujo-Menjouet, H. Rezaei, and L.M. Tine. Alzheimer's disease and prion: analysis of an in vitro mathematical model. Submitted, available at https://hal.archives-ouvertes.fr/hal-01708659/document.

D Craft. A Mathematical Model of the Impact of Novel Treatments on the A $\beta$ Burden in the Alzheimer's Brain, CSF and Plasma. Bull. Math. Biol., 64(5):1011-1031, 2002. ISSN 00928240.

H. Engler, J. Prüss, and G.F. Webb. Analysis of a model for the dynamics of prions II. J. Math. Anal. Appl., 324:98-117, 2006.

P.T. Francis, A.M. Palmer, M. Snape, and G.K. Wilcock. The cholinergic hypothesis of Alzheimers disease: a review of progress. Journal of Neurology, Neurosurgery \& Psychiatry, 66(2):137-147, 1999.

D.B. Freir, A.J. Nicoll, I. Klyubin, S. Panico, J.M. Mc Donald, E. Risse, E.A. Asante, M.A. Farrow, R.B. Sessions, H.R. Saibil, et al. Interaction between prion protein and toxic amyloid $\beta$ assemblies can be therapeutically targeted at multiple sites. Nature communications, 2:336, 2011.

P. Gabriel. The shape of the polymerization rate in the prion equation. Math. Comput. Model., 53(7-8):1451-1456, 2011. ISSN 08957177.

S.L. Gallion. Modeling amyloid-beta as homogeneous dodecamers and in complex with cellular prion protein. PloS one, 7(11):e49375, 2012.

D. A. Gimbel, H. B. Nygaard, E. E. Coffey, E. C. Gunther, J. Lauren, Z. A. Gimbel, and S. M. Strittmatter. Memory Impairment in Transgenic Alzheimer Mice Requires Cellular Prion Protein. J. Neurosci., 30(18):6367-6374, 2010. ISSN 0270-6474. 
M.L. Greer, L. Pujo-Menjouet, and G.F. Webb. A mathematical analysis of the dynamics of prion proliferation. J. Theor. Biol., 242(3):598-606, 2006. ISSN 0022-5193.

M. Helal, E. Hingant, L. Pujo-Menjouet, and G.F. Webb. Alzheimer's disease: analysis of a mathematical model incorporating the role of prions. J. Math. Biol., 69(5):1207-35, 2014. ISSN 1432-1416.

V.J. Hilser, J.O. Wrabl, and H.N. Motlagh. Structural and energetic basis of allostery. Annual review of biophysics, 41:585-609, 2012.

B. Iooss and P. Lemaître. A review on global sensitivity analysis methods. In Uncertainty Management in Simulation-Optimization of Complex Systems, pages 101-122. Springer, 2015.

C.R. Jr Jack, D.S. Knopman, W.J. Jagust, R.C. Petersen, M.W. Weiner, P.S. Aisen, L.M. Shaw, P. Vemuri, H.J. Wiste, S.D. Weigand, et al. Update on hypothetical model of Alzheimers disease biomarkers. Lancet neurology, 12(2):207, 2013.

G.B. James. The role of amyloid beta in the pathogenesis of Alzheimer's disease. J. Clin. Pathol., 66:362-366, 2013. ISSN 1472-4146.

R.D. Johnson, J.A. Schauerte, C. Chang, K.C. Wisser, J.C. Althaus, C.J.L. Carruthers, M.A. Sutton, D.G. Steel, and A. Gafni. Single-Molecule Imaging Reveals A $\beta 42$ :A $\beta 40$ Ratio-Dependent Oligomer Growth on Neuronal Processes. Biophys. J., 104(4):894-903, 2013. ISSN 00063495.

N. Kandel, T. Zheng, Q. Huo, and S.A. Tatulian. Membrane binding and pore formation by a cytotoxic fragment of amyloid $\beta$ peptide. The Journal of Physical Chemistry B, 121(45): 10293-10305, 2017.

E. Karran, M. Mercken, and B. De Strooper. The amyloid cascade hypothesis for Alzheimer's disease: an appraisal for the development of therapeutics. Nat. Rev. Drug Discov., 10(9): 698-712, 2011. ISSN 1474-1776.

H.W. Kessels, L.N. Nguyen, S. Nabavi, and R. Malinow. The prion protein as a receptor for amyloid- $\beta$. Nature, 466(7308):E3, 2010.

J. Laurén, D.A. Gimbel, H.B. Nygaard, J.W. Gilbert, and S.M. Strittmatter. Cellular prion protein mediates impairment of synaptic plasticity by amyloid- $\beta$ oligomers. Nature, 457(7233): 1128-1132, 2009. ISSN 0028-0836.

A. Lomakin, D.S. Chung, G.B. Benedek, D.A. Kirschner, and D.B. Teplow. On the nucleation and growth of amyloid beta-protein fibrils: detection of nuclei and quantitation of rate constants. Proceedings of the National Academy of Sciences, 93(3):1125-1129, 1996.

A. Lomakin, D.B. Teplow, D.A. Kirschner, and G.B. Benedek. Kinetic theory of fibrillogenesis of amyloid beta -protein. Proc. Natl. Acad. Sci. U. S. A., 94:7942-7947, 1997.

R.B. Maccioni, G. Farías, I. Morales, and L. Navarrete. The revitalized tau hypothesis on Alzheimer's disease. Archives of medical research, 41(3):226-231, 2010.

M. Nick, Y. Wu, N.W. Schmidt, S.B. Prusiner, J. Stöhr, and W.F. DeGrado. A long-lived a $\beta$ oligomer resistant to fibrillization. Biopolymers, 2018.

J. Nunan and D.H. Small. Regulation of APP cleavage by alpha-, beta- and gamma-secretases. FEBS Lett., 483(1):6-10, 2000. ISSN 00145793.

M. Prince, A. Wimo, M. Guerchet, G. Ali, Y. Wu, and M. Prina. World Alzheimer Report 2015 The Global Impact of Dementia. Alzheimer's Dis. Int., 2015.

J. Prüss, L. Pujo-Menjouet, G.F. Webb, and R. Zacher. Analysis of a model for the dynamics of prions. Discret. Contin. Dyn. Syst. Ser. B, 6(1):225-235, 2006.

S.A. Small and K. Duff. Linking A $\beta$ and tau in late-onset Alzheimer's disease: a dual pathway hypothesis. Neuron, 60(4):534-542, 2008.

A.L. Sosa-Ortiz, I. Acosta-Castillo, and M.J. Prince. Epidemiology of Dementias and Alzheimer's Disease. Arch. Med. Res., 43(8):600-608, 2012. ISSN 01884409.

B. Urbanc, L. Cruz, S.V. Buldyrev, S. Havlin, M.C. Irizarry, H.E. Stanley, and B.T. Hyman. Dynamics of Plaque Formation in Alzheimer's Disease. Biophys. J., 76:1330-1334, 1999. 
ISSN 0006-3495. 